\title{
Online Retrieval and Indexing of Images using Multi Feature Vectors
}

\author{
Yatin Kumar Agarwal, Dilkeshwar Pandey, Manoj Singhal
}

\begin{abstract}
In technology proliferated era of modern world, health care has witnessed huge developments. The cutting edge technologies have paved way for sophisticated and feature rich image processing in medical field using colour tomography and medical resonance imaging. The images obtained using radiological techniques can be stored in a database and the features and implications can be recorded in the database after the analysis of those images by physicians. These databases can be used in obtaining the meaningful analysis of the images obtained through radiology in rural areas of developing countries like India, where sophisticated medical facilities are a dream for many in developing nations. The dataset of images can be divided into training and testing set. Training set of data is utilized to obtain multi feature vectors based on Caffe. Caffe is used in this training with a focus on image recognition. The image feature is a simple image pattern based on which the description of image can be obtained. The features of an image are transformed to a vector space using computer vision algorithms. Moreover a framework has been evolved in this paper to extract the features from image using image descriptors-white box algorithms and neural nets-black box algorithms. We also present the pros and cons of our novel framework for online retrieval and indexing of images using multi feature vectors.
\end{abstract}

Keywords: Image processing, vectors, caffe, image descriptors, neural nets.

\section{INTRODUCTION}

In many applications of image processing, it is very much important to store images in a database. After storing in the database, it becomes necessary in many occasions to retrieve the images from database. For further processing or retrieval, mainly content based concept is employed but for indexing, most of the time concept based /description based or text based image indexing [1] is utilized. In text based image indexing keywords, description of images, captions or natural language text is used. In the image indexing methodologies, mostly a set of attributes of an image stored in the database [2] is used. In the second approach, an integrated feature extraction/object recognition subsystem was utilized. The third and relatively less used approach consists of image annotation [3]. Moreover, low level image features are utilized in the few of the image indexing approaches.

These image indexing techniques find widespread applications in the medical domain and health care sector, as huge data storage is available at low cost. Advancements in image acquisition techniques paved way for large sized

Yatin Kumar Agarwal, Research Scholar (AKTU), Lucknow, U.P, India.

(Email: yatinaga@gmail.com)

Dr. Dilkeshwar Pandey, Professor CSE, KIET, Ghaziabad, U.P, India (Email: dilkeshwar.pandey@kiet.edu)

Dr. Manoj Singhal, Professor IT, GNIOT, Greater Noida, U.P, India.
Revised Manuscript Received on September 10, 2019.

(Email: manojsinghal1010@gmail.com)

image datasets. Especially in large sized image datasets in healthcare sector, lot of image analysis needs to be carried out, to extract meaningful information. This medical domain is considered to be one of the main areas where Content Based Image Retrieval (CBIR) has found huge application.

After indexing of images and the storage of images in databases, image retrieval techniques are applied for selecting and displaying the matching images. One of the oldest techniques used in image retrieval is text based. In this text based retrieval of images, the keywords may be image name, date of addition, deletion and modification. The fundamental problems faced in text based image retrieval include certain intangible components such as feelings, emotions and multiple expressions, with homonyms and misspellings. One of the recently and widely used techniques in image retrieval in today's applications include CBIR.

This paper is organized into many sections. Section 2 of this paper deals with the corresponding literatures in the field of image indexing and retrieval. Section 3 discusses about the approach taken by us in extracting various features using multi vectors. Section 4 presents the experimental results of our approach in the context of precision and accuracy using WEKA and provides a comparative analysis with three other approaches that are widely used in healthcare. The final section of the paper deals with the conclusion of the paper.

\section{LITERATURE REVIEW}

This section of the paper will deal with the analysis of various approaches that were followed in the literature.

Messaoudi et al [4], discussed about medical image indexing based on the reports obtained from various experts. Their work concentrated on the removal of unavailability of expert medical facilities in rural and semi urban areas. This paper had proposed a kind of comments' summary keywords-based method. The comments based keywords are very relevant to the comments or annotations provided by physicians.

These keywords that are extracted provide robust image indexation. His approach proposed by the authors is referred as Terminology Extraction of Annotation (TEA) mixed approach.

R.Chbeir et al [5], proposed another efficient method for image indexing in medical application. The authors have addressed the spatial and evolutionary issues of images using different types of relations. This method is considered to be highly explanatory and reliable mechanism for indexing images. 
Pang $\mathrm{S}$ et al [6], proposed a novel biomedical image indexing and retrieval system based on preferential deep learning. The deep learning approach in this paper is used to extract the high level and compact features of various biomedical images. Multiple hidden layer based approach is used to capture substantial feature structures of images with high resolution.

Justin Kestelyn et al [7], in his blog post in cloudera discussed about a solution for storage and retrieval of medical imaging files using distributed Apache Hadoop cluster. This method was considered by the authors to be cost effective and high performance. The discussion in this blog post considered the industry standard of Picture Archiving and Communication Systems (PACS).

$\mathrm{R}$ Cornet et al [8], proposed an indexing method for medical images using hierarchical cluster structure and the LBP operator.

R.Zwiggelaar et al [9], discussed variants of local binary pattern descriptors to classify breast tissue which are widely used as texture descriptors for local feature extraction and analysed the classification results for the variants of local binary patterns such as classic LBP (Local Binary Pattern), ELBP (Elliptical Local Binary Pattern), Uniform ELBP, LDP (Local Directional Pattern) and M-ELBP (MeanELBP)

S.Jabeen et al [10], proposed a novel CBIR technique based on the visual words fusion of speeded-up robust features (SURF) and fast retina keypoint (FREAK) feature descriptors. SURF is a sparse descriptor whereas FREAK is a dense descriptor.

Z.Mehmood et al [11],introduced effective visual words fusion technique based on speeded-up robust features (SURF) and histograms of oriented gradients (HOG) feature descriptors. HOG is used to extract global features, whereas SURF is used for the extraction of local features.

M.Yousuf et al [12], introduced an effective novel technique to improve the performance of CBIR on the basis of visual words fusion of Scale-Invariant Feature Transform (SIFT) and local intensity order pattern (LIOP) descriptors.

\section{IMAGE ANALYSIS USING MULTI FEATURE VECTORS}

A unique and novel approach to process the images in the medical image databases is developed and tested. This approach utilized the multiple features of an image such as region, colour, size, texture, HSV (Hue, Saturation Value), shape and metadata. These multiple features are utilized in the image analysis to improve the precision and accuracy of image retrieval from the databases. The approach that is used by the authors in this paper consists of Master/Slave agent architecture. Every feature of an image will be processed and corresponding match score is calculated for close image matching. This activity will be performed by corresponding feature agent. These feature agents are a piece of software code that is capable of handling image processing in a distributed environment. All the scores from multiple agents belonging to multiple features will be handed over to a master feature agent in the database server. This master feature agent will compute the overall weighted score of the image features and the agent master extracts the

first ten most similar images to the end user of the system. The entire process is depicted in fig. 1 Shown below:

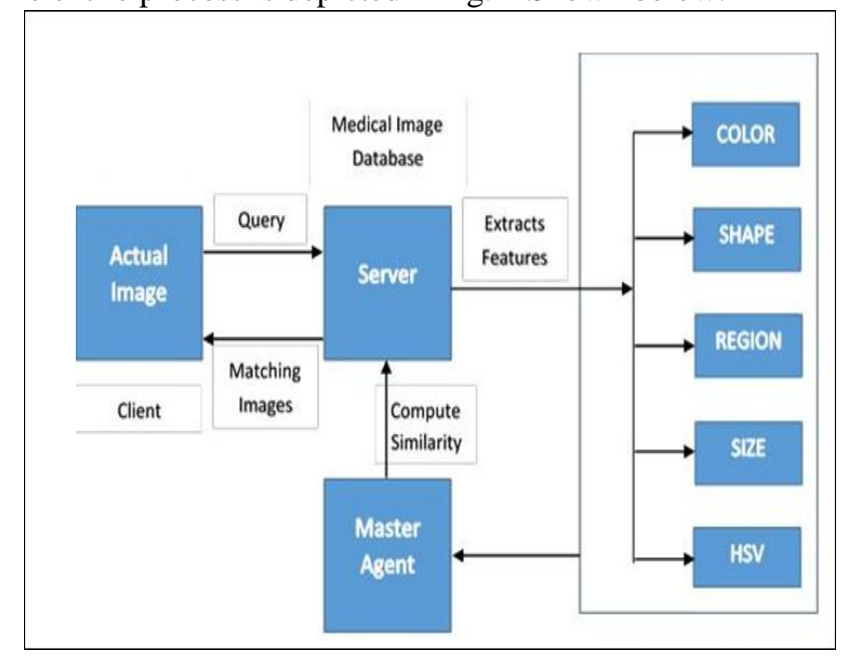

Fig.1: Novel Image Retrieval Architecture

Based on the architecture given in this section, we have simulated the processing framework on a sample medical image database and the results of which are discussed in the next section.

The master agent for calculation of consolidated scores performs better due to the matching algorithm that we implemented using Mahalanobis distance than Euclidean distance.

Mahalanobis distance always performs better than Euclidean distance because the multi feature attributes are taken together to calculate the consolidated similarity measure. Euclidean distance is calculated using the formula that contains attributes of the image in question and the attributes of the reference images are taken into consideration. Mahalanobis distance is calculated using the multiple attributes along with the covariance matrix calculated based on the multiple feature based attributes.

\section{EXPERIMENTAL RESULTS AND ANALYSIS}

The experimental setup used in the analysis of the proposed novel architecture of image processing consists of a medical image database that contains 1000 sample records. The slave agents for image feature extraction and corresponding score calculator for that particular feature were implemented in MATLAB [Reference number]. Fig.2 provides the picture of the agent processing that happens in the distributed environment. Also, after obtaining the consolidated scores of an image for similarity matching in the databases with the help of master agent, the accuracy and precision are calculated with the help training set and testing set data using WEKA data analysis tool. The proposed novel architecture (Master/Slave Agent Algorithm) have shown to produce better results than those feature based image retrieval techniques such as GLCM (Gray Level Co-Occurence Matrix), CCM (Colour CoOccurence Matrix) for different numbers of training set images such as 100, 250, 300, 500, 800 and 1000 . 


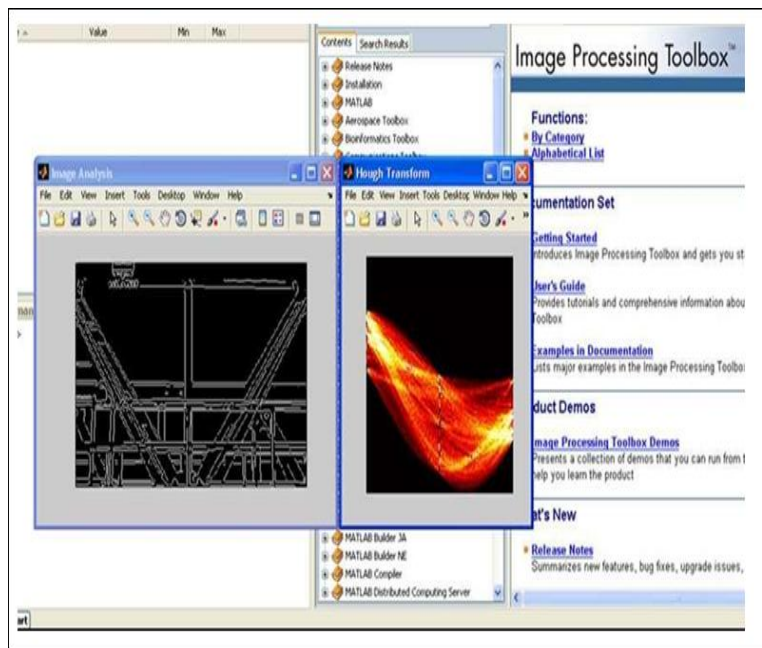

Fig.2: Agent Processing in MATLAB

Fig. 3 provides the performance of Master/Slave Agent Algorithm from the perspective accuracy and Fig.4 deals with precision in comparison with GLCM and CCM approaches.

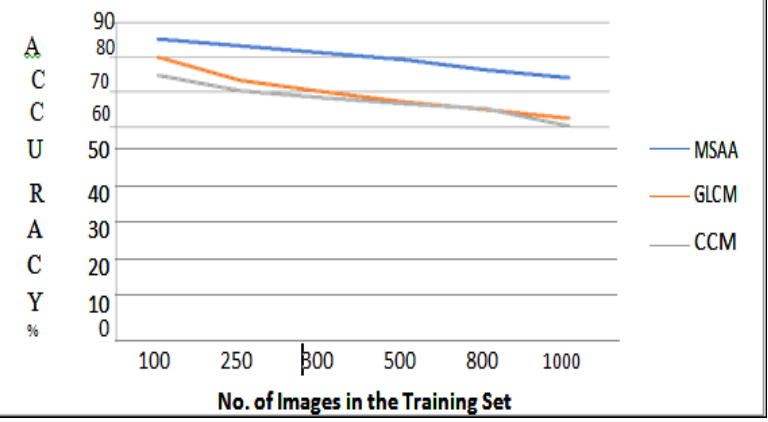

Fig.3: Accuracy of MSAA/GLCM/CCM approaches

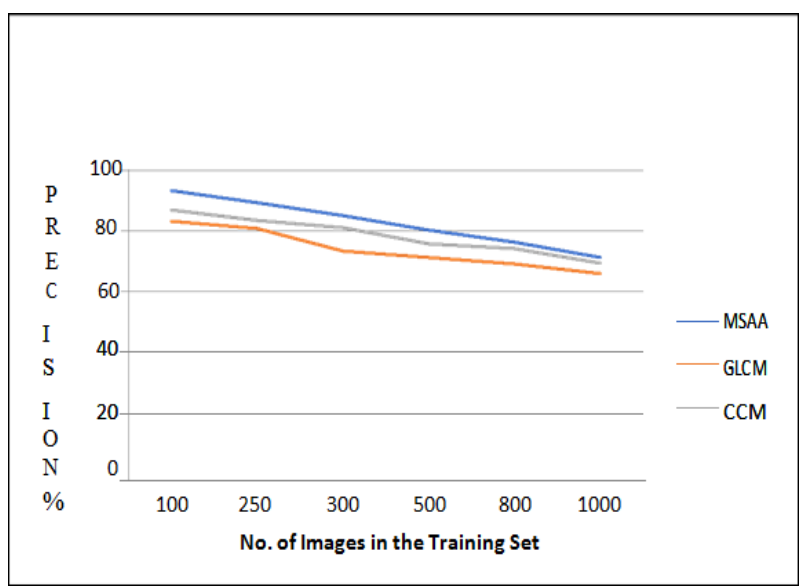

Fig.4: Precision of MSAA/GLCM/CCM approaches

\section{CONCLUSION}

This paper addressed a unique problem in today's healthcare industry, i.e., image indexing and retrieval from medical image databases. The paper utilized a novel approach in the form of the multi agent software techniques with master/slave architecture. Also instead of utilizing Euclidean distance, we have used Mahalanobis distance for efficient similarity score calculation using multi feature vectors of the images. These images are tested using WEKA tool for online retrieval towards the metrics of accuracy and precision. Accuracy and Precision of the proposed MSAA is compared against GLCM and CCM approaches and that it is concluded that our approach fared better in terms of different sets of training images in WEKA.

\section{REFERENCES}

1. Avinash N Bhute, B.B. Meshram, "Text Based Approach for Indexing and Retrieval of Image and Video: A Review", Advances in Vision Computing-An International Journal, March 2014.

2. G. M. Petrakis,"Image Representation, Indexing and Retrieval Based on Spatial Relationships and Properties of Objects", Ph.D dissertation, University of Crete, 1993.

3. Https://www.cogiotech.com/services/image-annotation

4. A. Messaoudi, R. Bouslimi, J. Akaichi, "Indexing Medical Images based on collaborative experts reports", International Journal of Computer Applications, May 2013.

5. S. Atnafu, R. Chbeir, and L. Brunie, "Content-based and metadata retrieval in medical image database". In Proceedings of the IEEE Symposium on ComputerBased Medical Systems, pages 327-332, 2002.

6. Pang S, "A novel biomedical image indexing and retrieval system via deep preference learning", COMPUT METHODS PROGRAMS BIOMED. 2018.

7. J. Kestelyn, "How to rocess and ndex Medical images with Apache Hadoop-Cloudera Engineering Blog", 2016.

8. R. Mukundan, "Image Features Based on Characteristic Curves and Local Binary Patterns for Automated HER2 Scoring”, Journal of Imaging, February 2018.

9. M. George, R. Zwiggelaar, "Comparative Study on Local Binary Patterns for Mammographic Density and Risk Scoring”, Journal of Imaging, February 2019. Safia Jabeen, Zahid Mehmood, Toqeer Mahmood, Tanzila Saba, Amjad Rehman, Muhammad Tariq Mahmood, "An effective content-based image retrieval technique for image visuals representation based on the bag-of-visualwords model", PLOS ONE, April 2018.

10. Zahid Mehmood, Fakhar Abbas, Toqeer Mahmood, Muhammad Arshad Javid, Amjad Rehman, Tabassam Nawaz, "Content-Based Image Retrieval Based on Visual Words Fusion Versus Features Fusion of Local and Global Features", Arabian Journal of Science and Engineering, December 2018.

11. Muhammad Yousuf, Zahid Mehmood, Hafiz Adnan Habib, Toqeer Mahmood, Tanzila Saba, Amjad Rehman, Muhammad Rashid, "A Novel Technique Based on Visual Words Fusion Analysis of Sparse Features for Effective Content-Based Image Retrieval", Mathematical Problems in Engineering, 2018. 